Risk factors for extra-ophthalmic involvement and treatment outcomes in patients with IgG4-related ophthalmic disease (see page 736)

Bilateral disease, high IgG4 level, and long symptom duration were associated with extra-ophthalmic involvement of IgG4-related ophthalmic disease. Combined immunosuppressant treatment was more effective in preventing relapse than prednisolone alone in patients with extra-ophthalmic involvement.

Keratopigmentation with micronised mineral pigments: complications and outcomes in a series of 234 eyes (see page 742)

Different techniques of keratopigmentation have been established with the use of various materials and pigments. Complications encountered by patients postoperatively have multifactorial aetiology and can be divided into organic and functional complications.

Comparison among adjuvant treatments for primary pterygium: a network metaanalysis (see page 748 )

The Netwok Meta-analysis comparing adjuvant treatments for primary resected pterygium has shown that the combination of conjunctival autograft and Cyclosporine A 0.05\% eye drops is the most effective in preventing recurrence.

Long-term follow-up of benign positional vertical opsoclonus in infants: retrospective cohort (see page 757)

Benign positional vertical opsoclonus, known as paroxysmal tonic downgaze, may be more common than reported, in otherwise healthy infants. Although it usually leads to thorough investigation, it resolves spontaneously with a very good outcome.

Should tumbling E go out of date in amblyopia screening? Evidence from a population-based sample normative in children aged 3-4 years (see page 761 ) This study provides reference data for tumbling $\mathrm{E}$ in 3-4 year old children. When compared with the existing literature, the tumbling $\mathrm{E}$ has a higher testability rates, especially in children of 3 years old.
Prevalence of amblyopia and its association with refraction in Chinese preschool children aged 36-48 months (see page 767 )

In a cross-sectional, population-based study conducted in children aged 36- to 48 months in Eastern China, manifest amblyopia was detected in $1.47 \%$ children, mainly associating with refractive error.

Asymmetrical accommodation in hyperopic anisometropic amblyopia (see page 772 )

Accommodation was investigated in anisometropic amblyopes using the PlusoptiX SO4 photorefractor. The majority (58\%) demonstrated aniso-accommodation. A unique finding of 'anti-accommodation' was present in 23\%. Only 19\% demonstrated symmetrical accommodation, refuting previous research.

Lens power in Iranian schoolchildren: a population-based study (see page 779)

A population based study of 6-12 year old schoolchildren described lens power by age, gender, place of residence, refractive errors and showed its related factors. The findings are useful for better understanding of lens power changes.

An evaluation of intracameral mydriasis for routine cataract surgery (see page 784)

Intracameral Mydrane provides adequate mydriasis in most patients undergoing cataract surgery. It may be associated with a positive patient experience but in this particular setting, was not judged to be a cost-effective intervention for routine use.

Outcomes of lensectomy and risk factors for failure in spherophakic eyes with secondary glaucoma (see page 790) Lensectomy was effective in controlling IOP in half of all eyes with spherophakia and glaucoma. Younger age ( $<6$ years), IOP $>32 \mathrm{~mm} \mathrm{Hg}$ and higher cup-to-disc ratio predicted need for longterm glaucoma medication or surgery.

Characteristics of early filtering blebs that predict successful trabeculectomy identified via three-dimensional anterior segment optical coherence tomography (see page 796)

The analysis of filtering blebs using sweptsource three-dimensional anterior segment optical coherence tomography indicates that a thick-walled, tall bleb, exhibiting the striping phenomenon at 2 weeks post-trabeculectomy, is a good prognostic factor for a successful trabeculectomy.

5 -year disease progression of patients across the glaucoma spectrum assessed by structural and functional tools (see page 802)

Determination of Glaucoma Progression requires both structural and functional tools, depending on disease severity. Though the absolute attenuation of RNFL thickness depended on that at baseline, the percentage loss appeared similar in all progressors.

Paediatric optic neuritis: factors leading to unfavourable outcome and relapses (see page 808 )

In 102 children optic neuritis, visual acuity recovery rate was $71 \%$, and visual field abnormalities persisted in 34\%. Age $\geq 10$, optic disc pallor and multiple sclerosis were associated with poor recovery $(\mathrm{p}<0.01)$.

Non-invasive method of monitoring retinal vasculitis in patients with birdshot chorioretinopathy using optical coherence tomography (see page 815 )

Retinal vasculitis is a potentially blinding condition that is typically monitored using fluorescein angiography. This study demonstrates that standard spectral domain OCT scans of the posterior pole can be used to non-invasively monitor large vessel retinal vasculitis activity in patients with birdshot chorioretinopathy.

Optical coherence tomography angiography reproducibility of lesion size measurements in neovascular age-related macular degeneration (AMD) (see page 821)

Optical Coherence Tomography Angiography (OCTA) was used to assess the reproducibility of choroidal neovascularization size showing a high intra-user and inter-user agreement(0.98 for both) for all types of neovascular membranes in age related macular degeneration.

Green emission fluorophores in eyes with atrophic age-related macular degeneration: a colour fundus autofluorescence pilot study (see page 827)

In eyes affected by age-related macular degeneration (AMD), short-wave 


\section{At a glance}

fluorophores are present in the macular atrophic area, and appear to correspond to residual debris or drusenoid material.

Predictors of radio-induced visual impairment after radiosurgery for uveal melanoma (see page 833 )

The authors report quantitative correlation between clinical and dosimetric variables and the risk pf the main side-effects in patients treated with Gamma Knife radiosurgery for uveal melanoma. Dose constraints could potentially reduce sideeffects onset.

$\mathrm{p} 16^{\mathrm{INK} 4 \mathrm{a}}$ overexpression as a predictor of survival in ocular surface squamous neoplasia (see page 840 )

p $16^{\text {INK4a }}$ immunoexpression and methylation status in 64 OSSN patients revealed that $\mathrm{p} 16^{\mathrm{INK} 4 \mathrm{a}}$ overexpression is a poor prognostic predictor in advanced AJCC stage. Sunlight induced promoter hypermethylation contributes towards pathogenesis of OSSN.
Immunohistochemical evaluation of stress responsive protein sestrin 2 and its correlation with p53 mutational status in eyelid sebaceous gland carcinoma (see page 848 )

P53 mutations were identified both in tumour and circulating cell free DNA samples. Downregulation of sestrin2 a downstream target of p53 was found be significantly associated with p53 gene mutations in eyelid sebaceous gland carcinoma. 